\title{
Sources of evidence: search engines
}

\author{
Jeyanthi John
}

Senior Researcher, Centre for Evidence-based Dentistry, Oxford, UK

\author{
Previously in this series I have described the major biomedical databases, Medline \\ and the Cochrane Library, and other biomedical sources. In this final article I briefly \\ outline a few of the other available sources of information on the Internet. \\ Evidence-Based Dentistry (2004) 5, 47-49. doi:10.1038/sj.ebd.6400247
}

The Internet is a source of an enormous amount of information on just about any subject you could think of. Search engines help to retrieve information selectively without your having to undertake the almost impossible task of trawling through countless websites looking for it. They use specialised computer programs that move through websites and send information back to a database. When a search term is entered, the search engine checks through this database and produces a list of 'hits'. Thousands of such hits may be produced for any search term and the list of hits is organised differently depending upon which search engine is used.

Meta-search engines search multiple databases instead of multiple websites. In theory, this would save you searching all these separate databases. In reality, the quality of the searches is still dependent on the quality of the databases these meta-search engines search, and how they organise the results. Most metasearch engines only display the top 10 to 100 results from each search engine they use. Many meta-search engines integrate the results, that is, merge duplicate findings, and rank the results according to particular criteria, such as relevance to search term. Here are some search engines and meta-search engines which you might find useful.

\section{Alta Vista (www.altavista.com)}

Alta Vista is a very user-friendly search engine. There is a search box for you to type in a search term or phrase. Search tabs above the search box allow search- ing in different areas: Web (the default option), Images, MP3/Audio, Video and News. It has a "translate" option, which lets you translate the page and also automatically translates all of the pages you link to from that page.

You can build a search query using a series of boxes that prompt you to type in words or phrases you would like the search engine to include or exclude. Additionally, it allows the user to search using the Boolean query search box and the use of connectors such as AND, OR, NOT and NEAR, just as in PubMed. You can specify a date-range, confine searching to a specific world region and limit the number of results from the same site using "site collapse". For new users, the "help" function is simple and informative. The websites found by AltaVista are not evaluated and it is left to the user to review and judge their content and relevance.

\section{Google (www.google.com)}

Google is currently the largest search engine currently available on the web. It evaluates sites according to how popular they are - a system called Page Ranking. This is estimated using various parameters including the number of links they have from other sites, for example, whether a site (such as Yahoo!) refers to that particular site. The more links a site has, therefore, the higher it is rated by Google. It is consequently less likely to pick up obscure pages such as personal homepages.

Google is very straightforward to use. Users just need to type a word or phrase into the search box and click on "Google search" which produces a series of hits. Clicking on the "I'm Feeling Lucky" option produces a website that Google identifies as closest to the word or phrase you have entered. As with AltaVista, a series of tabs above the search box allows you to search within the Web (the default option), Images, Groups, Directory or News.

An advanced search option allows you to build a query using a series of boxes to define the search for words or phrases. It will also limit the returned hits to a date range or format. A separate section allows you to search within a specific topic area, for example, Universities. There is even a facility to search for products on sale, from office equipment to toys.

You can restrict your searching to pages in a particular language or filter out unwanted material using the Preferences tab. There are also options to search in a specific language or translate pages using the "Language Tools" tab.

Overall, Google is a comprehensive search engine and is therefore widely used. It is worth remembering, however, that the contents of the pages have not been evaluated in any way. It is the responsibility of the user to review and judge the content and relevance of the websites that Google finds.

MetaCrawler (www.metacrawler.com) MetaCrawler was one of the earliest meta-search engines. It was originally developed in 1994 at the University of Washington but is now owned and operated by InfoSpace (InfoSpace Inc. Bellevue, Washington, USA). MetaCrawler searches the major search engines including Google, Yahoo!, AltaVista, Ask Jeeves, About, LookSmart, Overture and FindWhat. The search results are integrated and ranked according to relevance with summaries for each. 
Again, you can customise it using the "Preferences" option to the right of the search box. This enables you to set your preferred language, the number of results from each source and whether you want the results sorted according to relevance or search engine. The advanced search option allows users to include particular words or phrases, and supports Boolean searches. For new users, clicking on "Tools and Tips", next to the search box, will provide you with search tips about how to use MetaCrawler effectively. Selecting "About MetaCrawler" at the bottom of the page will give you a brief overview about the meta-search engine.

\section{BIOME (biome.ac.uk)}

BIOME (Figure 1) is a collection of subject gateways that provides access to resources in the health and life sciences. It is aimed at researchers, students and practitioners working in these fields. It was created by a team of information specialists and subject experts based at the University of Nottingham Greenfield Medical Library. The core team work with a number of other organisations within the fields of health and life sciences to evaluate resources for BIOME. The fact that the resources are evaluated is a major advantage of BIOME over other search engines such as Google.

Each subject gateway in BIOME allows the user to search in a particular area. For example, Organising Medical Networked Information, or OMNI, is the subject gateway for health and biomedicine. It can be accessed by clicking on "OMNI" in the left column on the BIOME website. OMNI can also be accessed directly via http://omni.ac.uk. Individual resources are evaluated with respect to the scope and intended audience, content and format. The evaluation process is described on the website, which also includes a discussion of how to evaluate Internet resources.

\section{SUMSearch (sumsearch.uthscsa.edu)}

SUMSearch (Figure 2) is a specialist medical search engine created by Professor Bob Badgett and Linda Levy of the University of Texas Health Science Centre at San Antonio. It was designed to make Internet literature searches for medical evidence simpler by automating the process.

SUMSearch searches the Internet much like a meta-search engine but also does

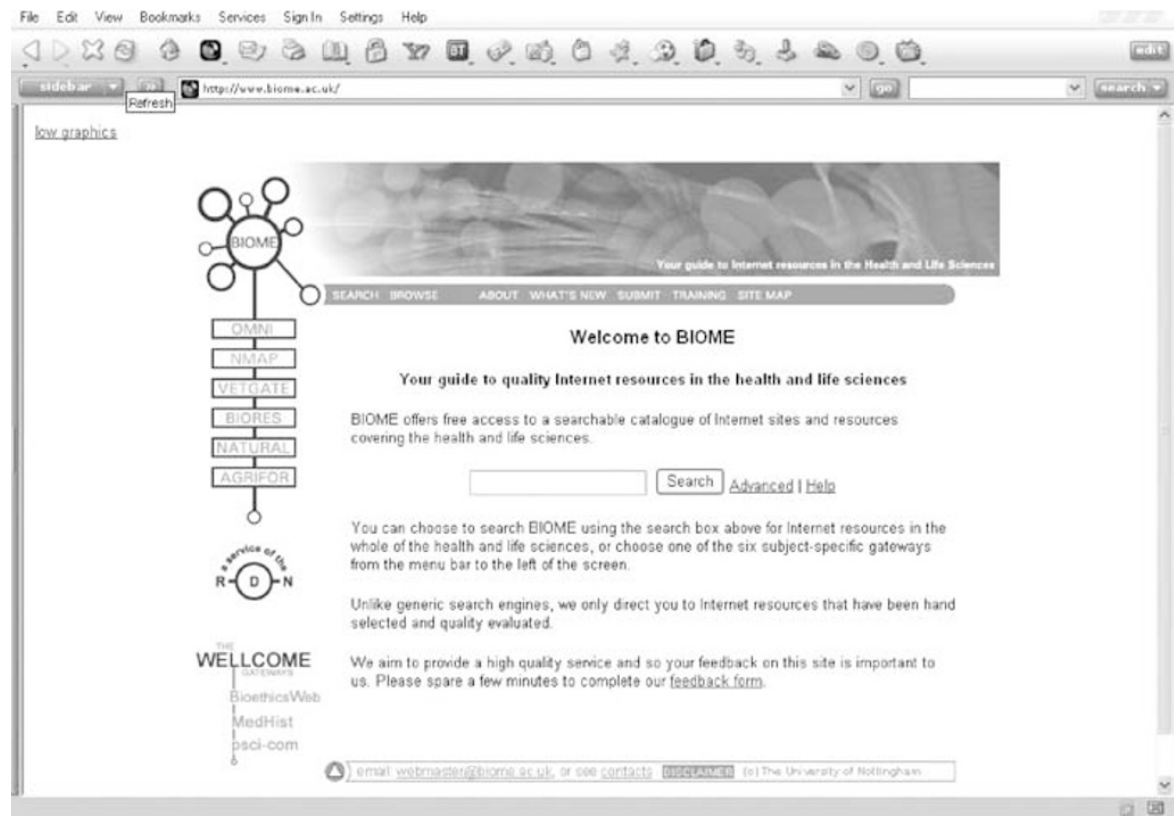

Figure 1.

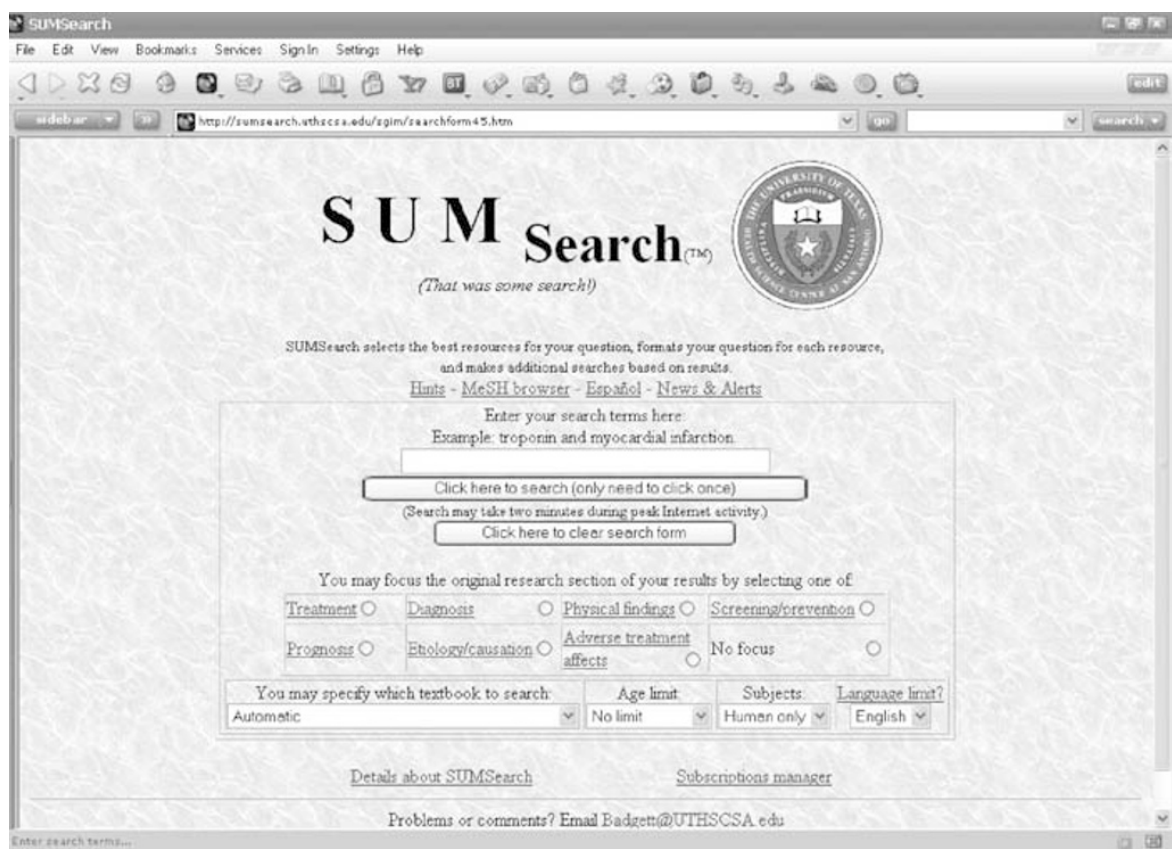

Figure 2.

so-called contingency searches. This means that if there are too many hits from a particular site, SUMSearch will do up to four additional searches until it finds an optimum number of hits. It may also search an additional site if there are few hits from a particular site.

The hits are arranged according to the breadth of discussion. Links to resources that provide a broader discussion, for example, textbooks, are at the top and those that provide a more focused discussion, for example, systematic reviews, are at the bottom. This makes it easy for the user to select the resources that best suit their query. Another advantage of SUMSearch is that it searches sites that have been deemed good-quality material by the SUMSearch team, for example, the National Library of Medicine and the 
Database of Abstracts of Reviews of Effectiveness. So, users have the security of knowing that the information is valid.

\section{TRIP (www.update-software.com/ scripts/clibNG/HTML/TRIPUsername- Logon.htm)}

The TRIP (Turning Research into Practice) database was developed in 1997 by Jon Brassey to help supply information to healthcare providers. It has developed and grown with much input from users.

The "Simple Search" in TRIP operates by searching for keyword matches on the document title only. Simple Boolean searching (AND, OR) is possible and "*”" can be used to specify words starting with particular letters. "dent*" will therefore look up dentist, dental and dentistry. The * is not a wildcard as other databases, such as PubMed, and cannot be used in the middle of a word. The results are displayed according to a list of categories including guidelines, general peer-reviewed journals, e-textbooks and patient information leaflets.

The advanced search option allows the above options plus the added ability to search for a word in the title only, or in the title or text. You can also restrict the search to a particular date range or to particular types of publications. TRIP keeps a search history record, like PubMed, which enables you to combine searches.

In April 2003, and enhanced version of TRIP, TRIP +, was created. TRIP + requires a subscription but is updated monthly and allows more enhanced searching.

\section{EviDents (medinformatics.uthscsa.edu/ EviDents)}

EviDents (Figure 3) is a search engine specifically developed for evidence-based dentistry. It was created by Richard Niederman, Director of the DSM-Forsyth Centre for Evidence-based Dentistry at the Forsyth Institute in Boston, USA. It is a straightforward programme with a series of boxes to help focus your search. Clicking on the title above each box pulls up a help screen, which explains what you could enter in the box. Selecting the "Hints" tab at the top of the screen produces a short series of tips. Selecting the "MeSH Browser" produces a search box, which you can use to look up a search term or phrase in the PubMed Browser.

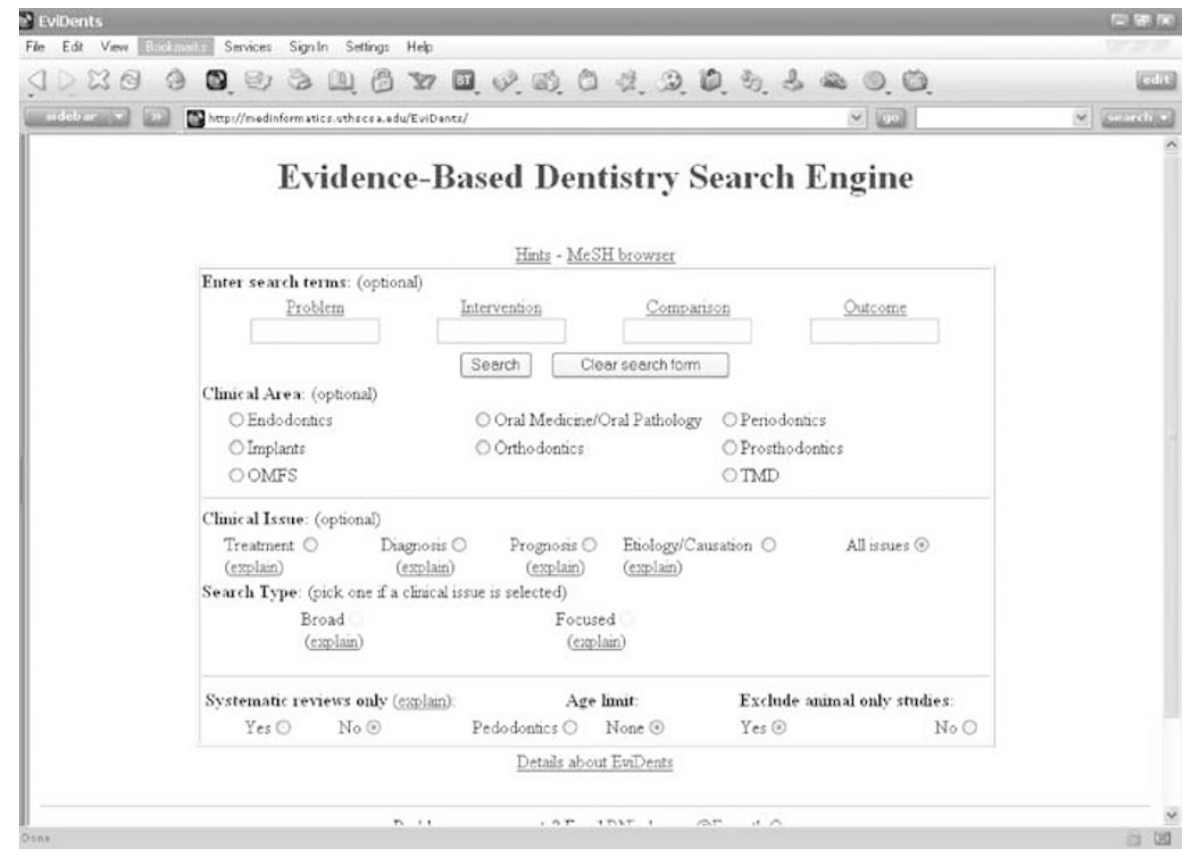

Figure 3.

The search page starts with a series of four boxes, which are designed using the PICO method of question formulation: Patient, Intervention, Comparison and Outcome. You may enter search terms into any or all of the four boxes. The next series of boxes that follows enables the user to refine the search according to clinical area, for example, periodontics, implants, etc. You can also specify the clinical issue, such as treatment, diagnosis, prognosis or etiology, or you can select all these issues. You can further choose to make your search broad, focused or confine it to systematic reviews; you can select only pedodontic papers and exclude animal studies.

\section{Using search engines}

The Internet is a rich source of information and search engines are very useful in retrieving what you require. The best way to find your way around search engines is to use them. It is a good idea to use one or two search engines regularly to get used to them. Much of the information on the Web is not evaluated in any way and it is up to the user to decide if the information on it is valid. Even those that claim to the written or evaluated by experts may be inaccurate, biased or outof-date.

The search engine BIOME contains some excellent information for new searchers. Clicking on "About" on the blue bar at the top of the screen brings up a page which describes BIOME. Selecting "Guidelines" and then "BIOME Evaluation Guidelines" under "Evaluation Guidelines" brings up a series of documents that the BIOME team use to select resources for their site. It is a useful starting point for anyone new to searching or those wishing to find out more about using the Internet to access information.

Those interested in more information about search engines and meta search engines may find the following websites interesting:

www.indiana.edu/ librcsd/search/meta. html

www.lib.berkeley.edu/TeachingLib/ Guides/Internet/MetaSearch.html www.searchenginewatch.com 